\title{
Creating New Strategies to Enhance Postpartum Health and Wellness
}

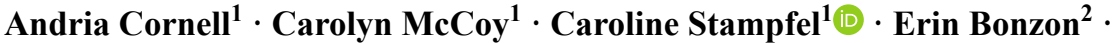 \\ Sarah Verbiest ${ }^{3}$
}

Published online: 31 August 2016

(C) Springer Science+Business Media New York 2016

\begin{abstract}
Over the past 5 years there have been a number of new initiatives focused on improving birth outcomes and reducing infant mortality, including a renewed focus on the complex interactions between motherhood and infancy that influence lifelong health trajectories. Beginning in 2012, the Association of Maternal \& Child Health Programs (AMCHP) facilitated a series of meetings to enhance coordination across initiatives. Emerging from these conversations was a shared desire across stakeholders to reimagine the postpartum visit and improve postpartum care and wellness. AMCHP convened a Postpartum ThinkTank Meeting in 2014 to map the system of postpartum care and identify levers for its transformation. The meeting findings are presented in an infographic which frames the challenges and proposed solutions from the woman's perspective. The infographic describes maternal issues and concerns along with a concise summary of the recommended solutions. Strategies include creating integrated services and seamless care transitions from preconception through postpartum and well-baby; business, community, and government support, including paid parental leave, health insurance and spaces for new parents to meet each other; and mother-centered care, including quality visits
\end{abstract}

Caroline Stampfel

cstampfel@amchp.org

1 Association of Maternal \& Child Health Programs, 2030 M St NW, Suite 350, Washington, DC 20036, USA

2 Zero to Three, 1255 23rd Street, NW, Suite 350, Washington, DC 20037, USA

3 UNC Center for Maternal and Infant Health, University of North Caroline School of Social Work, Old Clinic Building Room 3018, Campus Box 7181, Chapel Hill 27599-7181, USA on her schedule with complete and culturally appropriate information. These solutions catalyze a postpartum system of care that supports women, children, and families by infusing new ideas and capitalizing on existing opportunities and resources.

Keywords Postpartum - Birth outcomes ·

Maternal health $\cdot$ Title V $\cdot$ Systems integration

\section{Significance}

What is already known on this subject? Improving women's preconception and interconception health status can improve maternal and infant health. While being a new mother is a transformational and complex experience, approximately 20-30 percent of women do not even have a postpartum check-up within 6 weeks postpartum.

What this commentary adds? There is growing consensus that the postpartum period and 6-week check-up should be re-imagined to be inclusive of women's changing needs and interactions with various systems and supports. We present an infographic to guide the development of evidence and the implementation of mother-centered solutions.

\section{Background}

Over the past 5 years there have been a number of new initiatives and stakeholders focused on improving birth outcomes and reducing infant mortality. These initiatives, bolstered by growing integration of the life course approach in public health, reflect a shared urgency to act. Reducing infant mortality is a central goal of the Title V Maternal and Child Health (MCH) Services Block Grant Program and has been 
for over 80 years, since its passage in the Social Security Act of 1935. Despite significant progress in reducing infant death during that time frame, infant morbidity, maternal mortality and morbidity and persistent disparities by race and ethnicity demand further response. Recently, new partners have emerged to contribute their unique resources to this fight, creating a tapestry of public and private partners working at the national, state, and community levels to ensure that every baby born in the United States lives to his or her first birthday and fullest potential. Globally, infant mortality is an indicator of the overall health and wellbeing of women and girls. Following on the heels of national attention on infant mortality has been growing appreciation for improving women's preconception and postpartum health to ensure each mother has a healthy and safe pregnancy and childbirth experience to reduce the risk of maternal mortality and morbidity. Now more than ever, it is important to focus on the complex interactions between motherhood and infancy that influence lifelong health trajectories for both individuals.

\section{Emergence of Postpartum Care and Wellness as a Priority}

In recent years, the Association of Maternal \& Child Health Programs (AMCHP), with support from the W.K. Kellogg Foundation, proactively coordinated efforts to "connect the dots' across the numerous maternal and infant health initiatives to support collaboration, reduce duplication and accelerate progress. In response to requests from Title $\mathrm{V}$ program leaders across the country, in July 2013 AMCHP hosted a meeting of national and state leaders, funders, and partners to coordinate and prioritize efforts. One catalyst for this meeting was the new Health Resources and Services Administration's (HRSA) Maternal and Child Health Bureau's Collaborative Improvement and Innovation Network (CoIIN) to Reduce Infant Mortality. Five CoIIN priority areas to reduce infant mortality and improve birth outcomes emerged, including the expansion of access to preconception and interconception care. Throughout the 2-day meeting, the topic of postpartum care, including a call to reimagine the postpartum visit, stood out as a priority topic. Following the meeting, AMCHP and other national partner organizations recognized the need to take a closer look at the role of postpartum care and wellness in the United States in improving birth outcomes.

\section{Systems Mapping to Identify Solutions}

AMCHP subsequently hosted a national Postpartum ThinkTank meeting in December 2014 with the goal of illuminating the needs and solutions surrounding postpartum health and its link to better birth outcomes. Leaders from the National Maternal and Child Health (MCH) Workforce Development Center, housed in the Gillings School of Global Public Health at the University of North Carolina at Chapel Hill, taught national and state leaders and partners the fundamentals of system science terminology and methods, with a special emphasis on systems mapping and the development of a systems diagram. Participants also reviewed studies describing the barriers to postpartum care and wellbeing that women experience (Aber et al. 2013; Bennett et al. 2011, 2014; Coyle 2009; Declercq et al. 2013; Howell 2010; Martin et al. 2014). Meeting participants then broke into small groups by different stakeholders, including policymakers, payers, consumer advocates, public health, and clinicians. Each group identified key levers or opportunities that could be modified for positive downstream effects. The larger group then reconvened to combine all system maps into one large diagram. This new vision and system was centered on the collective concepts that the well-being of new mothers and families has value and that families should matter to a variety of stakeholders from business to policy makers and community leaders. This meeting highlighted the need to engage women and mothers as co-creators in developing a system of care that meets their needs. At the conclusion of the meeting, the group prioritized solutions and key strategies

\section{Opportunities for Improving Postpartum Systems of Care}

National experts and leaders identified three main directions for improving postpartum health and wellness including: (1) integrated services and seamless care transitions from preconception through postpartum and well-baby, (2) business, community, and government support, including paid parental leave, health insurance, and spaces for new parents to meet each other, and (3) mother-centered care, including quality visits on her schedule with complete and culturally appropriate information. These big picture solutions require buy-in from multiple sectors as well as detailed plans to operationalize the solutions at the community or state level.

For example, to focus on seamless care transitions, community stakeholders can explore opportunities to learn about the challenges women experience accessing postpartum care and services and mothers' ideas for action steps to enhance wellness. Leaders can begin to build relationships across sectors to simplify the steps women must take to access health care and needed services (e.g. postpartum clinic visit, family planning, WIC, behavioral health, etc.). Peer learning, such as through participation in CoIINs, is critical to advance innovation and build evidence-based practice. 
Further, to achieve business, community, and government support for new families, public health leaders should explore the kind of support employers need to better support new parents and families. Leaders in all sectors need to understand better how mothers connect with one another within and across communities, allowing businesses and communities to create spaces and places for new families to gather and access the support and services they need.

Work also must be done to define the content of postpartum care and build metrics to allow for assessment of the quality of care provided. Mother-centered care also hinges on the capacity of health professionals working in health care settings to provide culturally and linguistically appropriate services for the individuals they serve and how these settings are adapting their services and supports to the everyday lives of women. Sustained improvement in mother-centered care will also depend on how health systems learn whether women and families are satisfied with the care they receive as a component of quality.

\section{Call to Action}

The solutions posed through the system mapping exercise at the Think-Tank meeting point to actions all MCH stakeholders can take to mend a broken system of postpartum services. The infographic (see Fig. 1) is a concise, shareable, and visually compelling model that diverse stakeholders can use to prompt conversation. Concepts from studies about postpartum care noted above were phrased in the first person in the infographic to seed dialogue. These solutions capture a postpartum system of care that supports women, children, and families by infusing new ideas and capitalizing on existing opportunities and resources.

This is a critical time to make meaningful change for postpartum systems of care. The MCH community has a shared understanding of the influence of the postpartum period on the health of women, infants, and families, from mental and emotional health to breastfeeding to birth spacing and contraceptive use, and the period of tremendous transformation that it represents for women and families. Diverse sectors must work together to implement the best available science at all touchpoints with women during the postpartum period. The challenge ahead is to identify gaps in evidence, prioritize the most meaningful research questions, and engage communities, especially women, in designing, testing, and implementing solutions. Public health and in particular, Title V, has an important role to play in evaluating new approaches and translating findings into action to ultimately adapt services and supports around

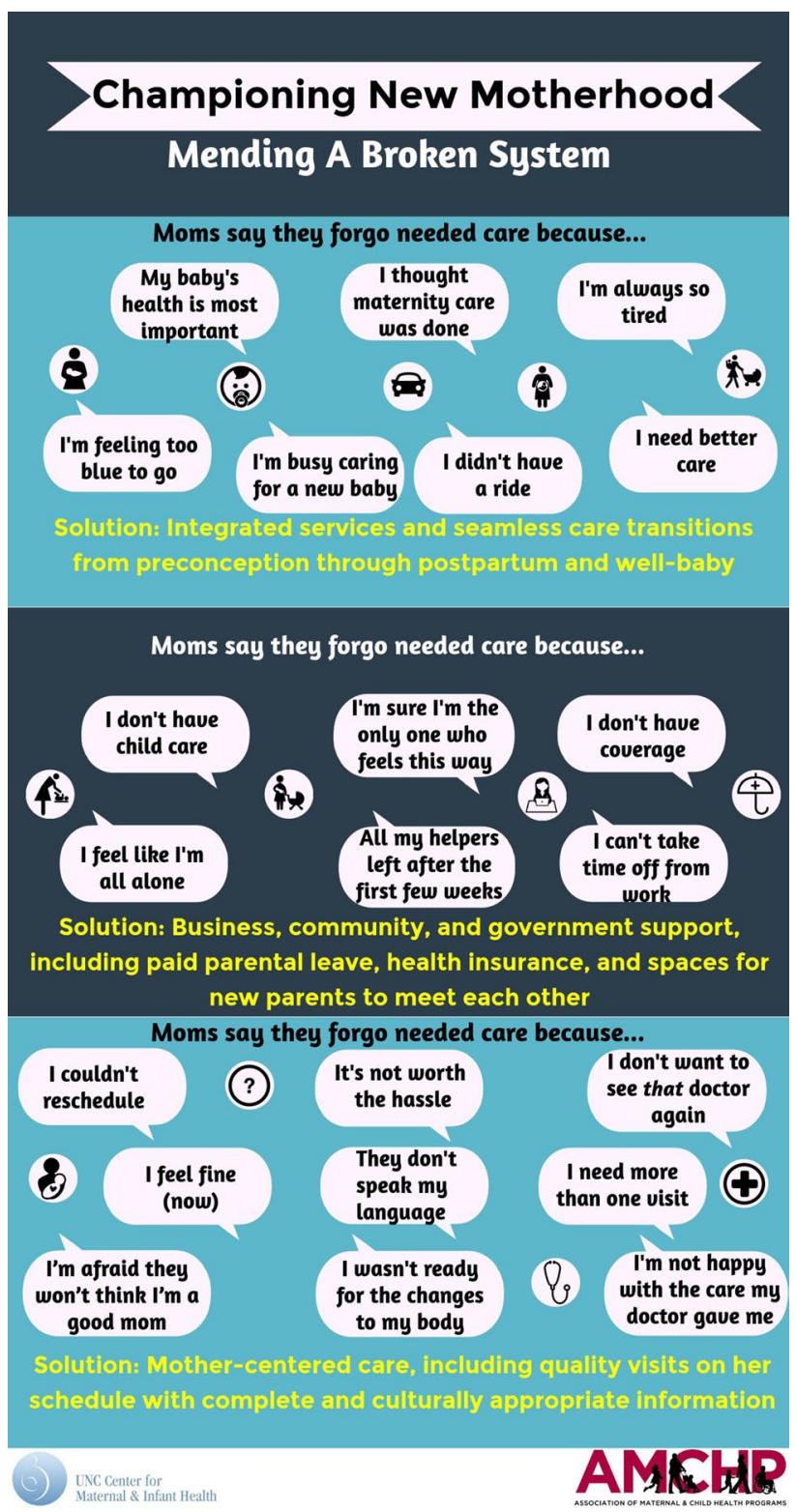

Fig. 1 Infographic of proposed solutions for improving systems of postpartum care

women and enable the postpartum period to be a facilitator to achieving lifelong wellness.

\section{About AMCHP}

AMCHP is the national membership association of state maternal and child health leaders. As an advocate, resource and partner, AMCHP's mission is to protect and promote the optimal health of women, children, and families. We envision a nation that values and invests in the health and wellbeing of all women, children, families, and communities. 


\section{References}

Aber, C., Weiss, M., \& Fawcett, J. (2013). Contemporary women's adaptation to motherhood the first 3 to 6 weeks postpartum. Nursing Science Quarterly, 26(4), 344-351.

Bennett, W. L., Chang, H. Y., Levine, D. M., Wang, L., Neale, D., Werner, E. F., \& Clark, J. M. (2014). Utilization of primary and obstetric care after medically complicated pregnancies: An analysis of medical claims data. Journal of General Internal Medicine, 29(4), 636-645.

Bennett, W. L., Ennen, C. S., Carrese, J. A., Hill-Briggs, F., Levine, D. M., Nicholson, W. K., \& Clark, J. M. (2011). Barriers to and facilitators of postpartum follow-up care in women with recent gestational diabetes mellitus: A qualitative study. Journal of Women's Health, 20(2), 239-245.
Coyle, S. B. (2009). Health-related quality of life of mothers: A review of the research. Health Care for Women International, 30(6), 484-506.

Declercq, E. R., Sakala, C., Corry, M. P., Applebaum, S., \& Herrlich, A. III (2013). Listening to Mothers III: Report of the third national US survey of women's childbearing experiences. New York: Childbirth Connection; 2013.

Howell, E. A. (2010). Lack of patient preparation for the postpartum period and patients' satisfaction with their obstetric clinicians. Obstetrics \& Gynecology, 115(2, Part 1), 284-289.

Martin, A., Horowitz, C., Balbierz, A., \& Howell, E. A. (2014). Views of women and clinicians on postpartum preparation and recovery. Maternal and Child Health Journal, 18(3), 707-713. 